URLs

ubiquitin http://ca.expasy.

org/uniprot/P62988

polı http://us.expasy.org/uniprot/Q9UNA4

Rev1 http://us.expasy.org/uniprot/Q9UBZ9

poln http://us.expasy.org/uniprot/Q9Y253

polк http://us.expasy.org/uniprot/Q9UBT6

PCNA http://us.expasy.org/ uniprot/P12004

DNA REPAIR

\title{
Family resemblance
}

Two novel ubiquitin-binding domains (UBDs) that are involved in translesion synthesis - the main pathway by which replication occurs across DNA lesions in mammalian cells - have been identified by Dikic and colleagues. As described in Science, their results indicate that these domains, which are evolutionarily conserved in all Y-family translesion-synthesis polymerases, have crucial regulatory roles in translesion synthesis.

The authors carried out yeast two-hybrid screens using wild-type ubiquitin and ubiquitin that was mutated at Ile44 (ubiquitin ${ }^{\star}$ ). All known UBDs interact with ubiquitin through its conserved Ile44 residue, so proteins that interact with ubiquitin* might contain novel UBDs. As the C terminus of translesion-synthesis polymerase $\mathrm{l}$ (polı) interacted with ubiquitin and ubiquitin*, it contains a UBD that does not require Ile44 to bind to ubiquitin.

Using bioinformatics approaches, the authors showed that the $\mathrm{C}$ terminus of polt contains two copies of a novel sequence, which they named the ubiquitin-binding motif (UBM). UBMs were also found in the Y-family polymerase Rev1. Mutating putative ubiquitin-binding residues in either of the polt UBMs reduced polt binding to ubiquitin, whereas mutating these residues in both UBMs abolished the interaction.

Dikic and co-workers identified a second novel UBD in their yeast two-hybrid screen. They named this UBD the ubiquitin-binding zinc finger (UBZ) domain, and it was found in the two remaining Y-family polymerases pol $\eta$ and polк. So, all of the Y-family polymerases have UBDs at their $\mathrm{C}$ termini.

Following DNA damage, the ubiquitylation of proliferating cell nuclear antigen (PCNA) directs the replication machinery into the translesionsynthesis pathway, which results in lesion bypass. However, how this modification of PCNA is recognized and interpreted by the cell has been unclear. Polt and pol $\eta$ colocalize with each other and with PCNA in replication factories, and this colocalization can be clearly seen following ultraviolet (UV) irradiation. The authors showed that this colocalization is dependent on the UBDs of these polymerases, because mutating the UBDs of polt and poln disrupted their localization.

Although polt and poln can bind to PCNA directly through their PCNA-interacting peptide (PIP) box, the authors showed that the DNA-damage-induced ubiquitylation of PCNA significantly improved the interaction between polt and ubiquitylated PCNA. The PIP box seems to bring specificity to the interaction, whereas the interaction between the UBMs and ubiquitin increases the avidity.

The authors next analysed the importance of the UBDs of poln in vivo, by using a fibroblast cell line that is defective in pol $\eta$ and sensitive to UV irradiation in the presence of caffeine. They showed that transfecting these cells with wildtype pol $\eta$ restored the sensitivity of these cells to that of normal fibroblasts, whereas transfecting these cells with UBZ-mutant pol $\eta$ had no such restoration effect. Transfecting these cells with PIP-box-mutant pol $\eta$ had only a minimal restoration effect, so both the ubiquitin- and the PCNAbinding abilities of pol $\eta$ are needed for efficient translesion synthesis.

In the final part of this work, Dikic and colleagues showed that polt and poln can themselves be monoubiquitylated, and this monoubiquitylation is dependent on their UBDs. Only the unmodified forms of poli and pol $\eta$ can interact with ubiquitin. The authors therefore propose that the inclusion of these polymerases in replication factories might be regulated by a cycling between their monoubiquitylated and unmodified forms.

Rachel Smallridge

ORIGINAL RESEARCH PAPER Bienko, M. et al. Ubiquitin-binding domains in $\mathrm{Y}$-family polymerases regulate translesion synthesis. Science 310, 1821-1824 (2005)

FURTHER READING Hicke, L., Schubert, H. L. \& Hill, C. P. Ubiquitinbinding domains. Nature Rev. Mol. Cell Biol. 6, 610-621 (2005) WEB SITE

Ivan Dikic's laboratory: http://molsig.biochem2.de/

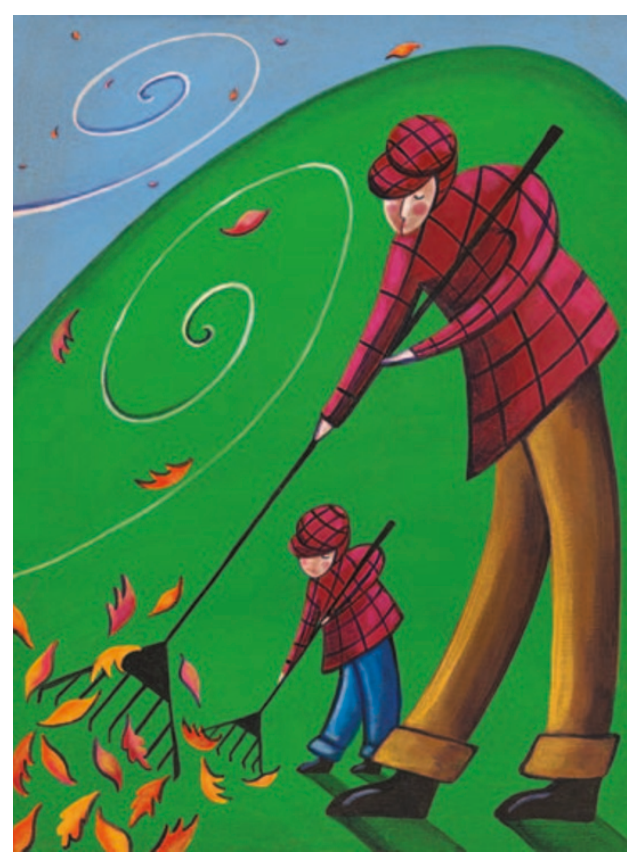

